\title{
Electron bunch length measurement with a wakefield radiation decelerator
}

\author{
Weiwei Li, Zhigang He, and Qika Jia \\ National Synchrotron Radiation Laboratory, University of Science and Technology of China, \\ Hefei, Anhui 230029, China
}

(Received 5 May 2013; published 31 March 2014)

\begin{abstract}
In this paper, we propose a novel method to measure the electron bunch length with a dielectric wakefield radiation (DWR) decelerator which is composed of two dielectric-lined waveguides (DLWs) and an electron spectrometer. When an electron beam passes through a DLW, the DWR is excited which leads to an energy loss of the electron beam. The energy loss is found to be largely dependent on the electron bunch length and can be easily measured by an electron spectrometer which is essential for a normal accelerator facility. Our study shows that this method has a high resolution and a great simplicity.
\end{abstract}

DOI: 10.1103/PhysRevSTAB.17.042801

PACS numbers: 29.27.Fh, 41.60.-m, 41.75.Ht

\section{INTRODUCTION}

There is growing interest in the generation, measurement and application of short electron bunches. Subpicosecond electron bunches are generally required to generate high power, coherent, $\mathrm{THz}$ radiation. Examples of powerful $\mathrm{THz}$ radiation include generation via synchrotron radiation [1,2], transition radiation [3], Smith-Purcell radiation [4] and wakefield radiation [5]. To provide high flux and short pulse x-ray beams, femtosecond electron bunches are required for inverse Compton scattering facilities [6]. For time-resolved $\mathrm{MeV}$ ultrafast electron diffraction facilities $[7,8]$, electron bunches are also needed to be as short as possible to achieve a high time resolution. X-ray free electron lasers (XFELs) also utilize short bunches with high charges to achieve an efficient lasing process in a single pass through the undulator [9]. Precise bunch length measurement methods are necessary for developing such kinds of facilities.

To measure the electron bunch length, many methods have been developed in the past decades. The streak camera has been widely used for the bunch length measurement, but the resolution is normally limited to $200 \mathrm{fs}$, and the camera is expensive. The rf zero phasing [10] and the rf deflecting [11,12] method have shown a few tens of fs temporal resolution. However, extra structures are needed in these two methods. The electro-optic sampling method has been developed enormously in the first five years of this century [13-16] and shown the ability of measuring subpicosecond bunches in a single shot, though it needs a fs laser pulse. Coherent synchrotron radiation [17], coherent transition radiation [18] and coherent diffraction

\footnotetext{
*Corresponding author. hezhg@ustc.edu.cn

Published by the American Physical Society under the terms of the Creative Commons Attribution 3.0 License. Further distribution of this work must maintain attribution to the author $(s)$ and the published article's title, journal citation, and DOI.
}

radiation $[19,20]$ have also been widely used in the electron bunch length measurement, nevertheless they generally measure the average bunch length in multishots and suffer from spectrum distortion problems. To determine the rms bunch length instead of the bunch shape, the coherent Smith-Purcell radiation method [21] and the coherent wakefield radiation method [22] have also been applied. Each of them can be a single-shot measurement after calibrations. However, they still need the radiation collector and detector elements.

As a relativistic electron bunch travels through the dielectric-lined waveguide (DLW), it drives coherent dielectric wakefield radiation that is confined to a discrete set of modes due to the waveguide boundaries. This slowwave structure supports modes with phase velocity equal to the beam velocity that are thus capable of efficient energy exchange with the beam. For these modes, the radiation energy is proportional to the DLW length and correlated to the electron bunch length. So, the bunch length can be measured by observing the frequency spectrum of the wakefield radiation, which is the wakefield radiation method [22] mentioned above. It uses different filters to pick out the radiation at different certain modes and a detector to measure the radiation power. However, the filters operate over specific bands for frequencies and the transmission of the radiation varies significantly with the frequencies within those bandwidths, making accurate measurements difficult. Besides, the radiation picked out by the filters may contain the wakefield radiation excited by the electron beam entering and leaving the DLW.

In this paper, we propose a new wakefield radiation based method to measure the electron bunch length. Two DLWs and an electron spectrometer are used in the method. The DLWs will be inserted into the beam transport line one by one, and the average beam energies can be measured by the electron spectrometer after the beams pass through the DLWs respectively. Comparing to the method in Ref. [22], two DLWs are used in the proposed scheme to avoid the problem of the wakefield radiation excited by the electron 
beam entering and leaving the DLW, and the filters and the $\mathrm{THz}$ detector are not needed in our scheme. The difference between these two measured beam energies can be regarded as the energy loss caused by the dielectric wakefield radiation (DWR). It is found that the energy loss is very sensitive to the bunch length. Furthermore, the shorter the bunch the greater the energy loss, which makes the method very suitable for the short bunch measurement. The features of the proposed method are: high resolution for electron bunches with high peak currents, reasonably simple and low cost (consider that the electron spectrometer is essential for a normal accelerator facility).

\section{PRINCIPLE OF DWR DECELERATOR}

Figure 1 shows the sketch map of the DLW, which is a hollow cylindrical dielectric tube coated on the outer surface with metal to form a dielectric-lined waveguide (DLW). The dispersion equation describing the transverse modes of the DLW structure, for the azimuthally symmetric $\mathrm{TM}_{0 n}$ case, is given by [23]

$\frac{I_{1} k_{0 n} r_{1}}{I_{0} k_{0 n} r_{1}}=\frac{\varepsilon_{r} k_{0 n}}{\kappa_{0 n}} \frac{J_{0}\left(\kappa_{0 n} r_{2}\right) Y_{1}\left(\kappa_{0 n} r_{1}\right)-Y_{0}\left(\kappa_{0 n} r_{2}\right) J_{1}\left(\kappa_{0 n} r_{1}\right)}{Y_{0}\left(\kappa_{0 n} r_{1}\right) J_{0}\left(\kappa_{0 n} r_{2}\right)-Y_{0}\left(\kappa_{0 n} r_{2}\right) J_{0}\left(\kappa_{0 n} r_{1}\right)}$,

where $k_{0 n}$ and $\kappa_{o n}$ are the radial wave numbers in the vacuum and dielectric regions, respectively, $\varepsilon_{r}$ is the relative permittivity of the dielectric material, and $n=$ $1,2,3, \ldots$ indexes the solutions to the transcendental equation. $J_{m}(x)$ and $Y_{m}(x)$ are Bessel functions of the first and second kinds of order $m$, and $I_{m}(x)$ is the modified Bessel function of the first kind.

When the electron beam passes through the DLW, the DWR is excited, which causes an energy loss of the electron beam that depends on the electron bunch length and can be easily measured by an electron spectrometer. The orthonormality relation between any two eigenmodes, the radiation power flow and energy for a certain $\mathrm{TM}_{0 n}$ mode can be written as [24]

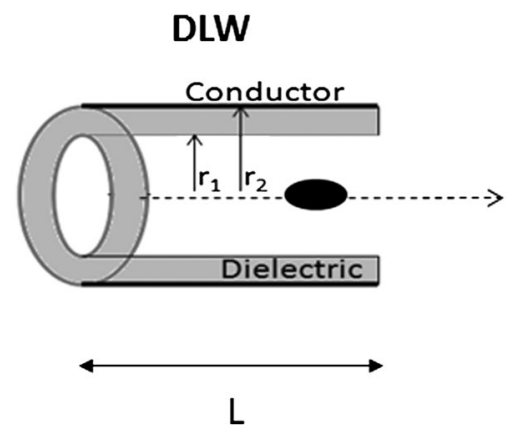

FIG. 1. The sketch map of the DLW.

$$
\begin{gathered}
\sum_{i=1}^{N=2} \int_{r_{i-1}}^{r_{i}} d r \cdot r\left[\varepsilon_{i} e_{z, m}(r) e_{z, n}(r)+\mu_{i} h_{z, m}(r) h_{z, n}(r)\right] \\
=C_{n} \delta_{m n}, \\
\overline{P_{0 n}}=-\beta c q_{0}^{2} \frac{e_{\frac{2}{2, n}(0)}\left(C_{n}\right.}{} \Theta(-s) \cdot \alpha_{n}{ }^{2}, \\
U_{0 n}=\frac{L}{\beta c} \cdot \overline{P_{0 n}},
\end{gathered}
$$

where $e_{z, m(n)}(r)$ and $h_{z, m(n)}(r)$ are the normalized eigenmode field components, $q_{0}$ is the charge,

$$
\Theta(-s)=\left\{\begin{array}{l}
1, s \geq 0 \\
0, s<0
\end{array}\right\},
$$

which means the radiation is excited behind the electron, and $\beta c$ is the velocity of the electron beam. $\alpha_{n}$ is the form factor and defined by

$$
\alpha_{n}=\left|\int_{-\infty}^{\infty} d s f(s) e^{-i k_{n} s}\right|,
$$

where $f(s)$ is the normalized longitudinal distribution function of the electron bunch. For a Gaussian bunch,

$$
\alpha_{n}=\exp \left(-\frac{\sigma_{z}^{2} k_{n}^{2}}{2}\right)
$$

where $\sigma_{z}$ is the rms bunch length. From these equations, we can find that the shorter the bunch the greater the energy loss of the electron beam. When the electron beam enters or leaves the DLW in a vacuum chamber, the wakefield radiation can also be excited by the electron beam passing from a pipe into another with a different diameter, but different from the DWR described above. That will be discussed in Sec. IV.

\section{DIMENSIONS OF DLW AND DEVIATION CAUSED BY FORM FACTOR}

For electron beams with different longitudinal distributions, the form factors are discrepant, thus the energy losses of electron beams are different. Figure 2 shows the form factors as a function of $\sigma_{z} k_{n}$ for three specific distributions. In order to apply the proposed DWR based method to the bunch length measurement, one needs to choose carefully the dimensions and material of the DLWs to obtain appropriate wave numbers $k_{n}$. First, the inner radius must be large enough for the beam transport. The dielectric tubes used in the scheme are drawn fused silica capillaries, a material chosen for its universal availability. The dielectric constant of the material is $\varepsilon_{r}=3.8$. The wave numbers $k_{n}$ (or frequencies) of the excited modes can be found from Eq. (1). For three different inner radii $r_{1}$, the scaling of these mode frequencies with outer radius $r_{2}$ is shown in Fig. 3. Because we expect this method can be used to measure the 


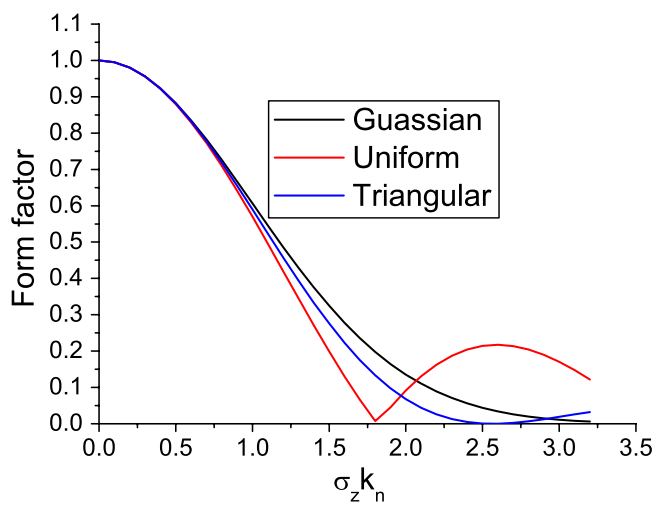

FIG. 2. The form factors as a function of $\sigma_{z} k_{n}$, the form factors are $\alpha_{n}=\exp \left(-\frac{\sigma_{z}^{2} k_{n}^{2}}{2}\right)$ (Gaussian), $\alpha_{n}=\frac{\sin \left(\sqrt{3} \sigma_{z} k_{n}\right)}{\sqrt{3} \sigma_{z} k_{n}}$ (uniform), and $\alpha_{n}=2 \frac{1-\cos \left(\sqrt{6} \sigma_{z} k_{n}\right)}{6 \sigma_{z}^{2} k_{n}^{2}}$ (triangular).

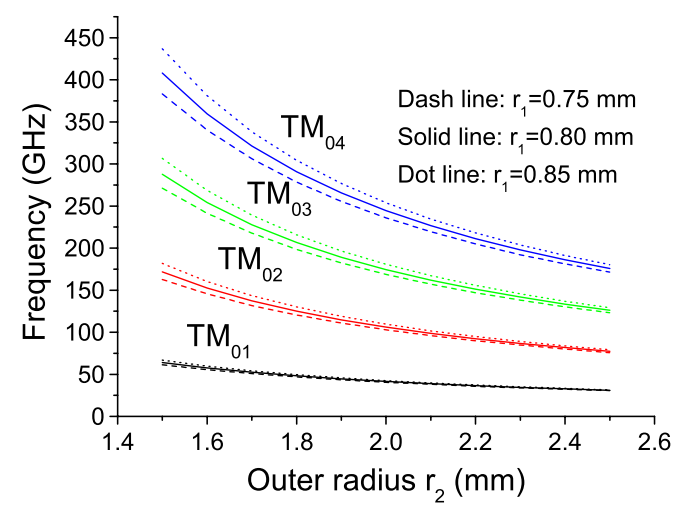

FIG. 3. Scaling of these mode frequencies with outer radius $r_{2}$, for three different inner radii $r_{1}$.

electron bunches in a wide dynamic range, the wavelength of the $\mathrm{TM}_{01}$ mode should be long enough to ensure that the wakefield radiation can be excited coherently by the electron beam even if the bunch length is relatively long. However, the coherence of the higher modes should be low to diminish the deviation caused by the form factor, which means the frequency separations between adjacent modes should be as large as possible. All things considered, the choices are $r_{1}=0.8 \mathrm{~mm}$ and $r_{2}=2.0 \mathrm{~mm}$ in this paper. It is worth mentioning that the chosen parameters are representative rather than fully optimized design set.

By using Eqs. (2) and (3), the power of the excited modes can be obtained, and the deviation of the energy loss caused by the form factors of electron bunches with different distributions can also be calculated. Figure 4 shows the relative power as a function of mode number $n$ for Gaussian electron beams with different lengths, and the values of $\sigma_{z} k_{n}$ are also listed in the caption.

For the three specific distributions used in Fig. 2, we can calculate that the deviations caused by the form factors for

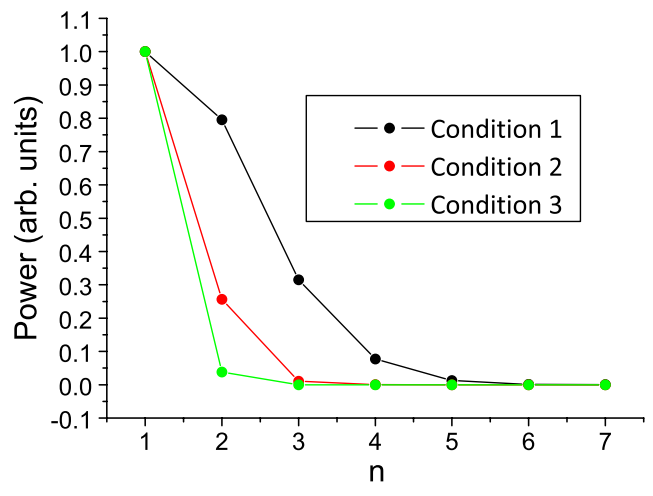

FIG. 4. The relative power of the excited $\mathrm{TM}_{0 n}$ modes. Condition 1: $\sigma_{z}=1 \mathrm{ps}, \sigma_{z} k_{1}=0.26, \sigma_{z} k_{2}=0.78, \sigma_{z} k_{3}=1.1$, $\sigma_{z} k_{4}=1.53$. Condition 2: $\sigma_{z}=2 \mathrm{ps}, \sigma_{z} k_{1}=0.52, \sigma_{z} k_{2}=1.34$. Condition 3: $\sigma_{z}=3 \mathrm{ps}, \sigma_{z} k_{1}=0.78$.

electron bunches with $\sigma_{z}=1 \mathrm{ps}$ and $\sigma_{z}=3 \mathrm{ps}$ are within $3.14 \%$ and $0.12 \%$, respectively.

\section{ANALYTICAL AND SIMULATED RESULTS}

The beam parameters, used in the analytical calculation and simulation of this paper (except for Secs. V Band V C), are listed in Table I.

We use the code XOOPIC [25] to simulate the coherent radiation and the energy loss of the electron beam. The simulation is calculated for the bunch passing from a larger diameter pipe through the DLW and back into the same larger diameter pipe. For a bunch with $1 \mathrm{nC}$ charge and $\sigma_{z}=1 \mathrm{ps}$, the energy loss as a function of the DLW length is shown in Fig. 5. The difference between the analytical and the simulated result is constant for different DLW lengths, which is caused by the wakefield radiation excited by the electron beam passing from a pipe into another with a different diameter. For a 2-cm-long DLW, the simulated on-axis longitudinal field is shown in Fig. 6(a). Figure 6(b) is a Fourier-transform result of the on-axis longitudinal field in Fig. 6(a). One can find that there is an obvious offset between the analytical and the simulated result, because only the DWR is considered in the analytical calculation. To compare the analytical and the simulated DWR spectrum result, we delete the longitudinal field components in the edge and interference zones according to Refs. [26-28], then the simulated DWR spectrum can be

TABLE I. Beam parameters used in the analytical calculation and the simulation.

\begin{tabular}{lc}
\hline \hline Beam energy $(\mathrm{E})$ & $100 \mathrm{MeV}$ \\
\hline Beam charge $\left(q_{0}\right)$ & 0.8 and $1.0 \mathrm{nC}$ \\
Transverse emittance $\left(\epsilon_{x, y}\right)$ & $2.5 \mu \mathrm{m}$ \\
rms bunch radius $\left(\sigma_{r}\right)$ & $200 \mu \mathrm{m}$ \\
rms bunch length $\left(\sigma_{z}\right)$ & 0.025 to $3.0 \mathrm{ps}$ \\
rms energy spread & $0.1 \%$ \\
\hline \hline
\end{tabular}




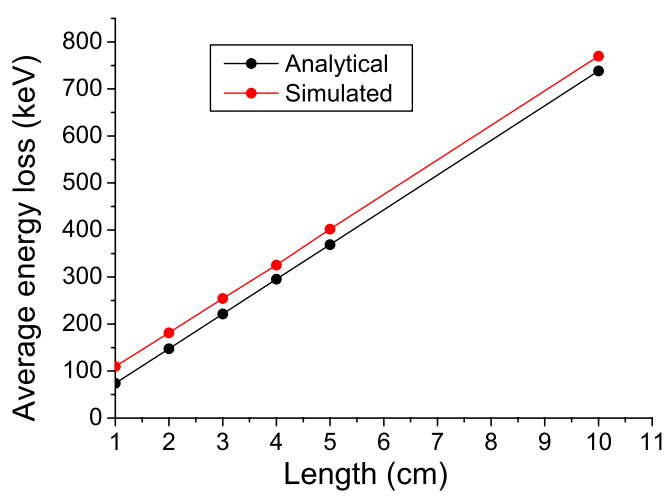

FIG. 5. Average energy loss caused by the wakefield radiation.


FIG. 6. (a) Longitudinal wakefield $E_{z}$ on tube axis versus position along tube; radiation spectra, calculated by Fourier transform, of (b) longitudinal wakefield $E_{z}$ along the whole tube and (c) longitudinal wakefield $E_{z}$ without its components in the edge and interference zones. obtained, as shown in Fig. 6(c). The analytical and the simulated result are basically coincident except for the scale deviations of the higher modes. Considering that the length scaling relation of the edge, interference and DWR zones is not critical, the deviations could come from the above processing procedure. The grid settings in the simulation and the Fourier-transform process could also bring deviations.

In general, the energy loss caused by the DWR can be analytically calculated, and the analytical result coincides with the simulated result. The wakefield radiation, excited by the electron beam passing from a pipe into another with a different diameter, is of particular interest. Although it cannot be analytically calculated at present, one can predict it using a simulation code. Alternatively, we can avoid this problem in the bunch length measurement of this paper. The measurement scheme will be shown in the next section.

\section{APPLICATION CONSIDERATIONS IN BUNCH LENGTH MEASUREMENT}

The sketch map of the bunch length measurement setup is shown in Fig. 7. A high precision motor should be used to control the positions of the DLWs. The electron spectrometer (composed of an analytic magnet and a measurement station) can be used to measure the average beam energy $E_{i}(i=1,2)$ after the beam passes through a DLW with $L_{i}(i=1,2)$ of length. " $E_{1}-E_{2}$ " can be regarded as the energy loss caused by the DWR in the length of " $L_{2}-L_{1}$ " with the beam passing through an infinite DLW.

\section{A. RESOLUTION}

The total energy loss caused by the DWR in the length of $L_{2}-L_{1}$ with the beam passing through an infinite DLW is

$$
U=\sum_{n=1}^{n} U_{0 n}=\sum_{n=1}^{n}-\frac{\left(L_{2}-L_{1}\right) q_{0}^{2} e_{2, n}^{2}(0)}{C_{n}} \Theta(-s) \cdot \alpha_{n}^{2}[J],
$$

where $U_{0 n}$ is given by Eq. (4). Converting the unit from $\mathrm{J}$ to $\mathrm{KeV}$, the average energy loss of electrons can be written as

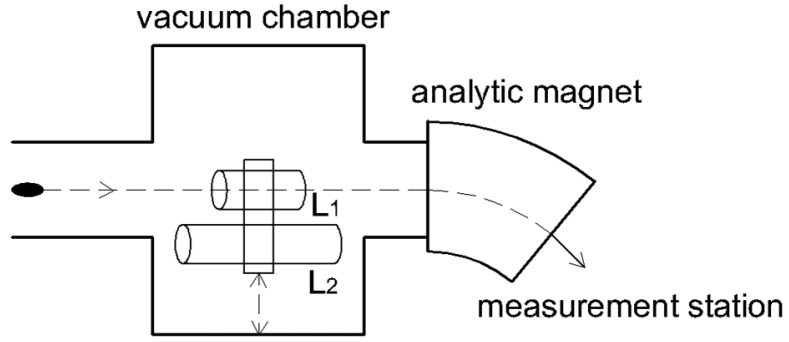

FIG. 7. The sketch map of the bunch length measurement setup. 


$$
\begin{aligned}
\mathrm{E}_{\mathrm{loss}} & =\frac{U}{q_{0} / e} \cdot \frac{1}{e}[\mathrm{eV}] \\
& =\sum_{n=1}^{n}-\frac{\left(L_{2}-L_{1}\right) q_{0} e_{z, n}^{2}(0)}{C_{n}} \Theta(-s) \cdot \alpha_{n}^{2} \times 10^{-3}[\mathrm{KeV}]
\end{aligned}
$$

Then, the resolution of the bunch length measurement apparatus can be defined by

$$
R=\frac{R_{s}}{\partial E_{\mathrm{loss}} / \partial \sigma_{z}}
$$

where $R_{s}$ is the resolution of the electron spectrometer. The resolution $R$ is proportional to the resolution of the spectrometer $R_{S}$, the bunch charge $q_{0}$ and the difference $L_{2}-L_{1}$.

For the DLWs with dimensions presented in Sec. III, the average energy loss as a function of rms bunch length is shown in Fig. 8. When the bunch length $\sigma_{z} \geq 200 \mathrm{fs}$, the shorter the bunch length the better the resolution. For electron bunches with $\sigma_{z}<100 \mathrm{fs}$, the resolution degrades dramatically.

To achieve a high resolution and make the measurement apparatus in a relatively small size, the difference $L_{2}-$ $L_{1}=20 \mathrm{~cm}$ is chosen. The parameters of the DLWs are listed in Table II. For measuring the average energy of electrons, we assume that the resolution of an electron spectrometer is $35 \mathrm{KeV}$. If the bunch charge is $q_{0}=1.0 \mathrm{nC}$, the resolutions for electron bunches within different ranges of length are listed in Table III. The resolution degrades to about 170 fs for a long electron bunch whose rms length is around $3 \mathrm{ps}$, nevertheless, it is better than that of the stateof-the-art streak camera ( $200 \mathrm{fs}$ ). To improve the resolution for electron bunches with lower charges, one can lengthen $L_{2}-L_{1}$ or improve the resolution of the electron spectrometer.

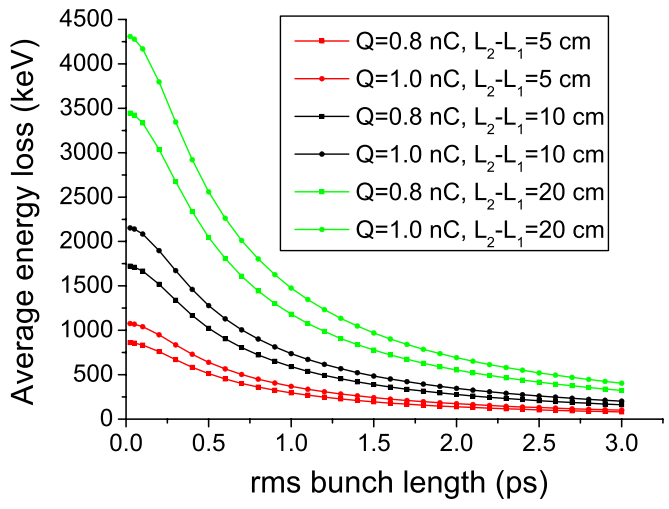

FIG. 8. Difference in average energy loss $\left(E_{2}-E_{1}\right)$ as a function of rms bunch length for a Gaussian bunch.
TABLE II. Parameters of the DLWs.

\begin{tabular}{lc}
\hline \hline Inner radius $\left(r_{1}\right)$ & $0.8 \mathrm{~mm}$ \\
\hline Outer radius $\left(r_{2}\right)$ & $2.0 \mathrm{~mm}$ \\
Dielectric constant $\left(\varepsilon_{r}\right)$ & 3.8 \\
Length of the short DLW $\left(L_{1}\right)$ & $1.0 \mathrm{~cm}$ \\
Length of the long DLW $\left(L_{2}\right)$ & $21.0 \mathrm{~cm}$ \\
\hline \hline
\end{tabular}

TABLE III. The resolutions for electron bunches with different ranges of length (the bunch charge is $1.0 \mathrm{nC}$ ).

\begin{tabular}{lc}
\hline \hline Range of bunch length $(\mathrm{ps})$ & Resolution (fs) \\
\hline 0.1 to 0.5 & 7.8 to 10.5 \\
0.5 to 1.0 & 10.5 to 25.0 \\
1.0 to 1.5 & 25.0 to 47.0 \\
1.5 to 2.0 & 47.0 to 78.5 \\
2.0 to 2.5 & 78.5 to 120.0 \\
2.5 to 3.0 & 120.0 to 170.0 \\
\hline \hline
\end{tabular}

\section{B. ERROR}

The analytical result of the deviation caused by the form factor has been presented in Sec. III. In this section, the machining error, the misalignment of the DLWs and the beam stability will be discussed.

The DLWs used in the proposed scheme can be custompulled hollow fused silica capillary tubes coated on the outer surface with metal. Considering the machining feature, the smoothness of the tube could be very well, and the inner and the outer radius of the tube can be measured to a very high precision. The measured frequency and energy of the radiation emitted from the DLW agreed quite well with the analytical results in Ref. [5], even though a very thin dielectric layer was used in their experiment. Furthermore, the deviation of the average energy loss of electrons is about $1.3 \%$ even if there is a $10 \mu \mathrm{m}$ thickness machining error along the whole tube, according to the analytical and the simulated result (see Fig. 3). Besides, the method of determining the eigenfrequencies of a DLW was also reported [29] and applied in the bunch length measurement experiment [22]. However, it is unnecessary in the proposed scheme of this paper.

There is always DLW misalignment in the experimental practice, which would cause a deviation of the centroid of the electron beam from the axis of the DLW. Table IV shows the simulation results of the errors caused by these deviations, where the beam energy is $100 \mathrm{MeV}$, the rms transverse size is $200 \mu \mathrm{m}$, the rms bunch length is $1 \mathrm{ps}$, and the bunch charge is $1 \mathrm{nC}$. For electron bunches with different energies and charges, the errors are identical. For an electron bunch with smaller transverse size, the error is larger. Even if the deviation is $50 \mu \mathrm{m}$, the error is within $1 \%$ for an electron bunch with a transverse size of $100 \mu \mathrm{m}$.

To accomplish the bunch length measurement, the average energies of two beams should be measured after 
TABLE IV. Simulated fractional errors caused by the deviations of the centroid of the electron beam from the axis of the DLW.

\begin{tabular}{lc}
\hline \hline Deviation $(\mu \mathrm{m})$ & Error $(\%)$ \\
\hline 10 & 0.29 \\
20 & 0.41 \\
30 & 0.52 \\
40 & 0.61 \\
50 & 0.68 \\
\hline \hline
\end{tabular}

they pass through the two DLWs respectively. So, the stabilities of the beam charge and energy should be considered. For a photoinjector, the fluctuation of the beam charge mainly comes from the fluctuation of the drive laser energy, and the fluctuation of the beam energy is caused by the timing jitter of the drive laser with respect to the rf wave. The measured rms energy stability of the drive laser and timing jitter are $1.2 \%$ and 0.16 ps respectively in the LCLS [11]. The rms error of the measured bunch length caused by the fluctuation of the drive laser energy will be $1.2 \%$. For the S-band $(2.856 \mathrm{GHz}) \mathrm{rf}$ accelerator, the phase jitter caused by the timing jitter $(0.16 \mathrm{ps})$ is about 0.16 degree, then the electron beam energy fluctuation $(\delta E)$ is within $15 \mathrm{KeV}$ for every $100 \mathrm{MeV}$ beam energy gain. The measurement error caused by the beam energy fluctuation is $\delta E /\left(E_{2}-E_{1}\right)$.

\section{REQUIREMENT OF THE BEAM ENERGY}

We should point out that this measurement system should be used in an accelerator facility with relatively high energy to avoid the potential transverse distortion of the electron beam caused by the radial field in the DLW and the possible nonlinear energy loss in the DWR zone of the DLW.

For a $\sigma_{z}=1 \mathrm{ps}$ electron bunch with $q_{0}=1 \mathrm{nC}$, the simulated evolution of the transverse beam size is shown in Fig. 9. If the beam energy is $50 \mathrm{MeV}$ and the initial beam size is $200 \mu \mathrm{m}$ at the entrance of the DLW, the transverse beam size will increase to $230 \mu \mathrm{m}$ at the exit of the DLW.

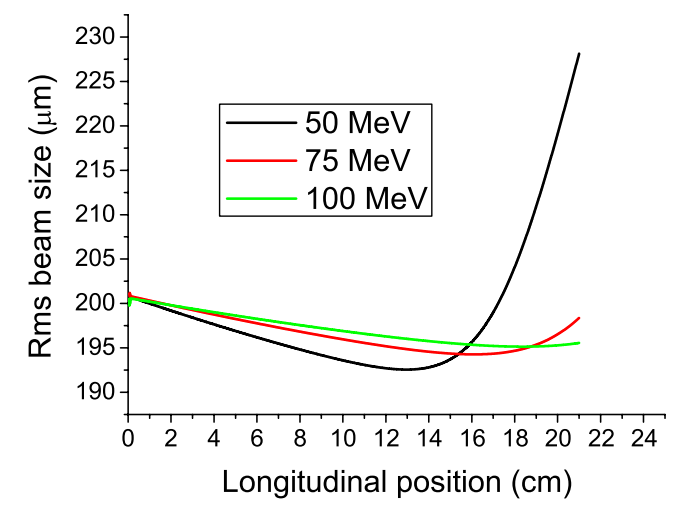

FIG. 9. Root-mean-square transverse beam size evolution along the longitudinal position of the DLW.
The bunch can pass the DLW without electron loss, since the inner radius of the DLW is $800 \mu \mathrm{m}$. When the beam energy is higher than $75 \mathrm{MeV}$, the transverse beam size is smaller than the initial $200 \mu \mathrm{m}$ along the whole $21 \mathrm{~cm}$ long DLW.

If the beam energy is relatively low, the velocity of the electron can change considerably because of the great energy loss along the DLW. Then, the energy loss is a nonlinear process according to Eq. (4). For electron bunches within energies of $50 \pm 4 \mathrm{MeV}$, the difference of the velocities is within $10^{-5}$, which is negligible. In summary, the proposed method can be used to measure the length of the electron bunch with energy higher than $50 \mathrm{MeV}$.

\section{APPLICATION EXAMPLE}

In Fig. 10, there is a beam line layout of a photoinjector. The gun is a 1.6 cell Brookhaven National Laboratory type photocathode rf gun, and the accelerators are 6 meters long, each of which consists of two 3-meters-long S-band (2.856 GHz) $2 / 3 \pi$-mode traveling-wave accelerators. For the harmonic compensation, an X-band (11.424 GHz) accelerator is located upstream of the bunch compressor (BC). The two beam position monitors are used for the beam trajectory angle determination and the beam collimation. The bend magnet is used for the electron spectrometer and the bunch charge measurement with a Faraday cup. At an upstream location of the bunch length measurement apparatus, there is an added current transformer (CT) for the bunch charge measurement. The Faraday cup and the CT are used to check the bunch charge before and after the beam passes through the DLWs. The bunch length measurement apparatus is composed of two DLWs, which are controlled by a high precision motor (as shown in Fig. 7). The parameters of the DLWs are listed in Table II. The two DLWs are inserted into the beam transport line one by one, and the average beam energies are measured after the beams pass through the DLWs respectively.

According to the simulation result, the rms bunch length is compressed to $0.72 \mathrm{ps}$ by the $\mathrm{BC}$, the bunch charge is $0.8 \mathrm{nC}$, the beam energy is $235 \mathrm{MeV}$ and the rms transverse beam size is $170 \mu \mathrm{m}$. To measure the average energy, the designed resolution of the electron spectrometer is $35 \mathrm{KeV}$ (which can be better). As to these parameters, the resolution of the bunch length measurement is around $20 \mathrm{fs}$. The measurement error will be within $5.1 \%$, which is the square

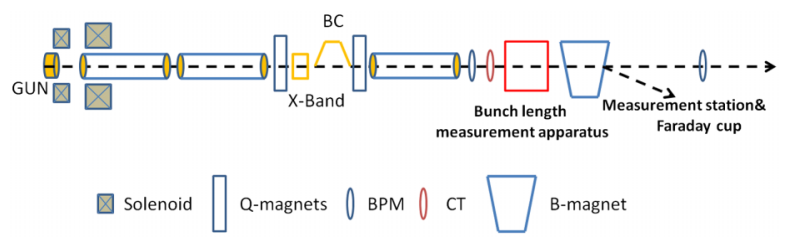

FIG. 10. Beam line layout of a photoinjector. 
root of the sum of the squares of the four individual errors: $3.5 \%$ caused by the form factor (see Sec. III, the compressed bunch is a quasi-Gaussian beam according to the simulation, and the actual error could be much smaller than this value), $1.3 \%$ caused by the machining error (see the second paragraph of Part B of Sec. V), $1 \%$ caused by the misalignment (see the third paragraph of Part B of Sec. V), and $3.4 \%$ caused by the beam stability problems (see the fourth paragraph of Sec. V B). As shown in Fig. 3, when the deviations of the inner and the outer radius are within $10 \mu \mathrm{m}$, the frequency differences are negligible for loworder modes which contain the most radiation energy, thus the change of the error caused by the form factor can be negligible, so these four individual errors can be treated as uncorrelated.

\section{SUMMARY AND DISCUSSION}

A new DWR based method is proposed for the electron bunch length measurement in this paper, which is composed of two DLWs with different lengths and a downstream spectrometer. The two DLWs will be inserted into the beam transport line one by one, and the average beam energies can be measured after the beams pass through the DLWs respectively. It is found that the difference between the average energies is very sensitive to the electron bunch length. Detailed investigations show that this method can be used to measure electron bunches within $\sigma_{z}=$ 0.1-3.0 ps and has a very high resolution for measuring short bunches with high charges. The DLWs can be installed in a vacuum chamber and do not need calibrations, therefore the proposed method is reasonably simple and can be easily implemented. The measurement apparatus is free of optical elements and the electron spectrometer is an essential for a normal accelerator facility, so the cost is relatively low. The possible errors of the method are also studied thoroughly and an application example is presented. It should be mentioned that recently the waveguide with a thin dielectric layer was offered as well for the beam energy measurement [30]. Thus, we can believe that this structure is suitable for versatile diagnostics of bunches.

The resolution of the measurement apparatus is proportional to the resolution of the spectrometer $R_{s}$, the bunch charge $q_{0}$ and the difference $L_{2}-L_{1}$. Although the resolution can be improved by lengthening the difference $L_{2}-L_{1}$ and improving the resolution of the electron spectrometer for the low charge beam, the proposed method is more suitable for electron bunches with relatively high charges $(>0.5 \mathrm{nC})$. Other disadvantages of the method are that it measures the bunch length in two shots rather than a single shot and it is a destructive one.

In addition to measuring the rms bunch length, the proposed method has the potentiality to determine the bunch shape. The longitudinal electric field of the selfwake at $r=0$ is



FIG. 11. Longitudinal electric field along the beam position for different bunch lengths $\sigma_{z}$.

$$
V^{-}(s)=-2 q_{0} \sum_{n=1}^{\infty} \frac{e_{z, n}^{2}(r=0)}{C_{n}} \int_{s}^{\infty} f\left(s^{\prime}\right) e^{i k_{n}\left(s-s^{\prime}\right)} d s^{\prime} .
$$

It can cause an energy modulation along the beam position. If the energy modulation can be measured, the longitudinal distribution function $f(s)$ is determined. The principle of this bunch shape measurement method is similar to that of the rf zero-phasing method. In order to measure the energy modulation along the beam position by the electron spectrometer, the intrinsic energy spread of the beam should be negligible comparing to the energy modulation caused by the longitudinal electric field, and the longitudinal electric field should be exclusive at different beam positions. To meet these requirements, the overall length of the electron beam should be shorter than a quarter of the wavelength of the $\mathrm{TM}_{01}$ mode, and the wavelength of the higher modes should be as short as possible to make their power negligible comparing to the power of the $\mathrm{TM}_{01}$ mode. For uniform temporal electron bunches with $q_{0}=1.0 \mathrm{nC}$, the longitudinal electric field (the first four higher-order modes are taken into account) along the beam position is shown in Fig. 11 for different bunch lengths $\sigma_{z}$, where the inner and the outer radius are 5.0 and $5.1 \mathrm{~mm}$, respectively. The frequencies of the first four higher-order modes are $0.1090,0.9095,1.7987$, and $2.6923 \mathrm{THz}$, respectively. With a meters-long DLW, the bunch shape can be measured to a high resolution. Considering the large radius and the long length of the DLW, the wakefield radiation, excited by electron beam passing from a pipe into another with a different diameter, can be negligible. However, the dynamic range of this method is narrow, and it is limited to measuring the electron bunch with high peak current (short bunch length and high charge).

\section{ACKNOWLEDGMENTS}

This work is supported by Chinese National Foundation of Natural Sciences under Contracts No. 11205152 
and No. 11375199, Fundamental Research Funds for the Central Universities under Contract No. WK2310000042, and Major State Basic Research Development Program of China under Grant No. 2011CB808301.

[1] G. L. Carr, M. C. Martin, W. R. McKinney, K. Jordan, G. R. Neil, and G. P. Williams, Nature (London) 420, 153 (2002).

[2] M. Abo-Bakr, J. Feikes, K. Holldack, P. Kuske, W. B. Peatman, U. Schade, G. Wüstefeld, and H.-W. Hübers, Phys. Rev. Lett. 90, 094801 (2003).

[3] W. P. Leemans et al., Phys. Rev. Lett. 91, 074802 (2003).

[4] S. E. Korbly, A. S. Kesar, J. R. Sirigiri, and R. J. Temkin, Phys. Rev. Lett. 94, 054803 (2005).

[5] A. M. Cook, R. Tikhoplav, S. Y. Tochitsky, G. Travish, O. B. Williams, and J. B. Rosenzweig, Phys. Rev. Lett. 103, 095003 (2009).

[6] R. W. Schoenlein, W. P. Leemans, A. H. Chin, P. Volfbeyn, T. E. Golver, P. Balling, M. Zolotorev, K.-J. Kim, S. Chattopadhyay, and C. V. Shank, Science 274, 236 (1996).

[7] H. Ihee, V. A. Lobastov, U. M. Gomez, B. M. Goodson, R. Srinivasan, C.-Y. Ruan, and A. H. Zewail, Science 291, 458 (2001).

[8] J. B. Hastings, F. M. Rudakov, D. H. Dowell, J. F. Schmerge, J. D. Cardoza, J. M. Castro, S. M. Gierman, H. Loos, and P. M. Weber, Appl. Phys. Lett. 89, 184109 (2006).

[9] LCLS Conceptual Design Report No. SLAC-R-593, 2002.

[10] D. X. Wang, G. A. Krafft, and C. K. Sinclair, Phys. Rev. E 57, 2283 (1998).

[11] R. Akre et al., Phys. Rev. ST Accel. Beams 11, 030703 (2008).

[12] J. Shi, H. Chen, C. Tang, Y. Du, W. Huang, L. Yan, R. Li, Q. Du, and D. Li, in Proceedings of the 23rd Particle Accelerator Conference, Vancouver, Canada, 2009 (IEEE, Piscataway, NJ, 2009), p. 3429.

[13] X. Yan, A. M. MacLeod, W. A. Gillespie, G. M. H. Knippels, D. Oepts, A. F. G. van der Meer, and W. Seidel, Phys. Rev. Lett. 85, 3404 (2000).
[14] I. Wilke, A. M. MacLeod, W. A. Gillespie, G. Berden, G. M. H. Knippels, and A. F. G. van der Meer, Phys. Rev. Lett. 88, 124801 (2002).

[15] G. Berden, S. P. Jamison, A. M. MacLeod, W. A. Gillespie, B. Redlich, and A. F. G. van der Meer, Phys. Rev. Lett. 93, 114802 (2004).

[16] A. L. Cavalieri et al., Phys. Rev. Lett. 94, 114801 (2005).

[17] R. Lai, U. Happek, and A. J. Sievers, Phys. Rev. E 50, R4294 (1994).

[18] H. C. Lihn, P. Kung, C. Settakorn, H. Wiedemann, and D. Bocek, Phys. Rev. E 53, 6413 (1996).

[19] M. Castellano, V. A. Verzilov, L. Catani, A. Cianchi, G. Orlandi, and M. Geitz, Phys. Rev. E 63, 056501 (2001).

[20] D. Xiang, X.-F. Yang, W.-H. Huang, C.-X. Tang, Y.-Z. Lin, W.-H. Li, Q. Pan, and M. Li, Chin. Phys. Lett. 25, 2440 (2008).

[21] S. E. Korbly, A. S. Kesar, R. J. Temkin, and J. H. Brownell, Phys. Rev. ST Accel. Beams 9, 022802 (2006).

[22] S. V. Shchelkunov, T. C. Marshall, J. L. Hirshfield, and M. A. LaPointe, Phys. Rev. ST Accel. Beams 8, 062801 (2005).

[23] T.-B. Zhang, T. Marshall, and J. Hirshfield, IEEE Trans. Plasma Sci. 26, 787 (1998).

[24] S. Y. Park and J. L. Hirshfield, Phys. Rev. E 62, 1266 (2000).

[25] J. P. Verboncoeur, A. B. Langdon, and N. T. Gladd, Comput. Phys. Commun. 87, 199 (1995).

[26] Changbiao Wang, J. L. Hirshfield, J. M. Fang, and T. C. Marshall, Phys. Rev. ST Accel. Beams 7, 051301 (2004).

[27] J. H. Kim, J. Han, M. Yoon, and S. Y. Park, Phys. Rev. ST Accel. Beams 13, 071302 (2010).

[28] I. N. Onishchenko, D. Yu. Sidorenko, and G. V. Sotnikov, Phys. Rev. E 65, 066501 (2002).

[29] J.-M. Fang, T. C. Marshall, J. L. Hirshfield, M. A. LaPointe, T.-B. Zhang, and X. J. Wang, in Proceedings of the 1999 Particle Accelerator Conference, edited by A. Luccio and W. MacKay (IEEE, New York, 1999), Vol. 5, p. 3627.

[30] A. V. Tyukhtin, Phys. Rev. ST Accel. Beams 15, 102801 (2012). 